\title{
Off-Resonance Excitation in a Linear Ion Trap
}

\author{
James W. Hager \\ MDS Analytical Technologies, Concord, Ontario, Canada
}

Off-resonance excitation coupled with mass-selective axial ejection of ions in a linear ion trap is shown to allow coherent control of a trapped ion population. Oscillations of the detected ion current have been found to correspond to the degree of detuning of the excitation field from the resonance frequency. Under appropriate excitation conditions coherent oscillations at the excitation frequency are seen that evolve into the ions' secular frequency on termination of the excitation field. Termination of the excitation field at various points during the off-resonance excitation profile leaves the ions with different degrees of radial excitation. The degree of radial excitation can be controlled by the coherent excitation field and is demonstrated to be useful for collision-induced dissociation. (J Am Soc Mass Spectrom 2009, 20, 443-450) (c) 2009 American Society for Mass Spectrometry

$\mathrm{O}$ ff-resonance excitation is used as a method for collisional excitation of ions in an ion cyclotron resonance (ICR) cell [1, 2]. Trapped ions are excited in a periodic manner at a beat frequency $\left(\omega_{\text {beat }}\right)$ corresponding to the difference between the ion's cyclotron frequency $\left(\omega_{c}\right)$ and the excitation frequency $\left(\omega_{\text {excite }}\right)$ :

$$
\omega_{\text {beat }}=\left|\omega_{c}-\omega_{\text {excite }}\right|
$$

Given the relatively low-pressure of the ICR cell environment, many cycles of re-excitation are required to impart sufficient internal energy for fragmentation. The sustained off-resonance irradiation (SORI) collisioninduced dissociation (CID) process based on this principle is a well-entrenched tool as a Fourier-transform (FT)-ICR ion dissociation technique [1].

Off-resonance excitation leading to fragmentation has also been demonstrated in three-dimensional resonant frequency (RF) ion traps at moderate pressures. Qin and Chait $[2,3]$ published results from "red-shifted off-resonance large-amplitude excitation" (RSORLAE) using very high excitation amplitudes $\left(\sim 21 \mathrm{~V}_{\mathrm{p}-\mathrm{p}}\right)$ that were red-shifted (i.e., shifted to lower frequencies) by about $5 \%$ of the RF voltage. This approach provided higher fragmentation efficiencies $(\sim 2.5 \times)$ for a variety of peptides [2]. It was noted that when the off-resonance excitation frequency was blue-shifted either no fragmentation was observed or the precursor ions were completely ejected from the ion trap. The model proposed focused on the "stretched" geometry of the three-dimensional ion trap and the associated higherorder multipole fields induced by this geometry change [2]. These higher-order fields lead to a blue shift of the frequency of characteristic ion motion as a function of radial amplitude. This blue shift moves the ion's secular

Address reprint requests to Dr. James Hager, MDS Analytical Technologies, Research, 71 Four Valley Drive, Concord, Ontario, Canada L4K 4V8. E-mail: jim.hager@sciex.com frequency away from the excitation frequency in the RSORLAE process, which would lead to a reduction in the maximum excursion of ion motion. Blue-shifted offresonance excitation is inefficient because the trapped ion's resonance frequency moves toward the off-resonance excitation frequency, which leads to further excitation and drives the ion more quickly toward the trapping electrodes and neutralization [2].

The present study extends off-resonance excitation to a low-pressure linear ion trap environment. Excitation is accomplished by detuning the excitation field a few hertz to several kiloherz to either the high- or lowfrequency side of the secular frequency of the trapped ions. The off-resonantly excited ions then gain radial amplitude in a periodic fashion at a frequency corresponding to the difference between the ions' secular frequency and that of the excitation field. The effectiveness of mass-selective axial ejection (MSAE) from a linear ion trap is strongly dependent on the radial amplitude of the trapped ions [4]. Thus, when the DC barrier at the exit end of the linear ion trap is sufficiently low, the off-resonantly excited ions are ejected axially toward the detector. Because of the periodic radial amplitude, axial ion ejection during nonresonant excitation yields a detected ion current with clear modulations at the beat frequency.

Higher-frequency modulations at the excitation frequency can also be observed. When the driving field is terminated, this modulation reverts to the secular frequency and is clearly visible on the detected ion signal. Experimental observation of ion motion at secular and RF frequencies has been reported previously following coherent excitation using either laser radiation [5] or short DC pulses [6, 7]. These earlier studies have provided insight into the dynamics of collisional processing within the three-dimensional ion traps as well as asymmetries in ion populations following photoionization. However, those experiments were either labo- 
rious or required significant geometry changes to the ion traps to allow entry of the laser light.

Off-resonance excitation leading to fragmentation can also be observed by maintaining appropriately high repulsive barriers at the ends of the linear ion trap. Higher barriers to exit can lead to further radial excitation during off-resonant excitation, resulting in ion activation and fragmentation or neutralization on the linear ion trap electrodes. Ion activation during offresonance excitation yields product-ion generation that also follows the same temporal profile of the beat frequency, since the laboratory frame kinetic energy changes periodically. Ideally, in the absence of ion loss processes such as fragmentation, ejection, and neutralization, the off-resonantly excited ions undergo repetitive excitation and de-excitation at the beat frequency. Once the excitation field is turned off, however, this changes. The phase at which the excitation field is terminated determines the fate of the excited ions. If the excitation field is stopped when the ions are at a minimum in their radial excursion, they remain in a state with little radial excitation. If the excitation field stops when the ions are near a maximum in radial amplitude, and thus kinetic energy, they maintain this degree of excitation, which can lead to additional fragmentation.

\section{Experimental}

A schematic of the modified 4000QTRAP hybrid quadrupole-linear ion trap mass spectrometer $(\mathrm{AB} /$ MDS SCIEX, Concord, Ontario, Canada) used in these studies is shown in Figure 1. The instrument is of a $\mathrm{Q}-\mathrm{q}-\mathrm{Q}_{\text {linear ion trap design }}$ and is based on a triple quadrupole ion path with the final quadrupole able to operate as a conventional RF/DC mass filter or as a linear ion trap with MSAE. The quadrupoles were driven by 0.816 $\mathrm{MHz}$ RF power supplies. Auxiliary dipolar AC voltages for ion excitation and mass analysis were provided by an external arbitrary waveform generator (Model 33220A, Agilent Technologies, Palo Alto, CA, USA). The arbitrary waveform generator was not phaselocked to the drive RF field.

The waveform generator was triggered to provide either an amplitude-modulated voltage at the appropriate frequency or triggered in "burst mode." Burst-mode operation provides a fixed-phase waveform at each trigger signal. The waveforms generated by amplitude modulation have random start phases. The difference between these two modes of operation becomes important when the temporal profiles of the ejected ion signals are averaged. If the excitation source has a random start phase, signal averaging will wash out any phase information associated with the excitation field. If the excitation source has a fixed start phase, then any phase specificity of the excitation field will be preserved. The exact phase chosen for waveform generator in burst mode has been found to be unimportant in that it leads to only a small positional shift of the higher-
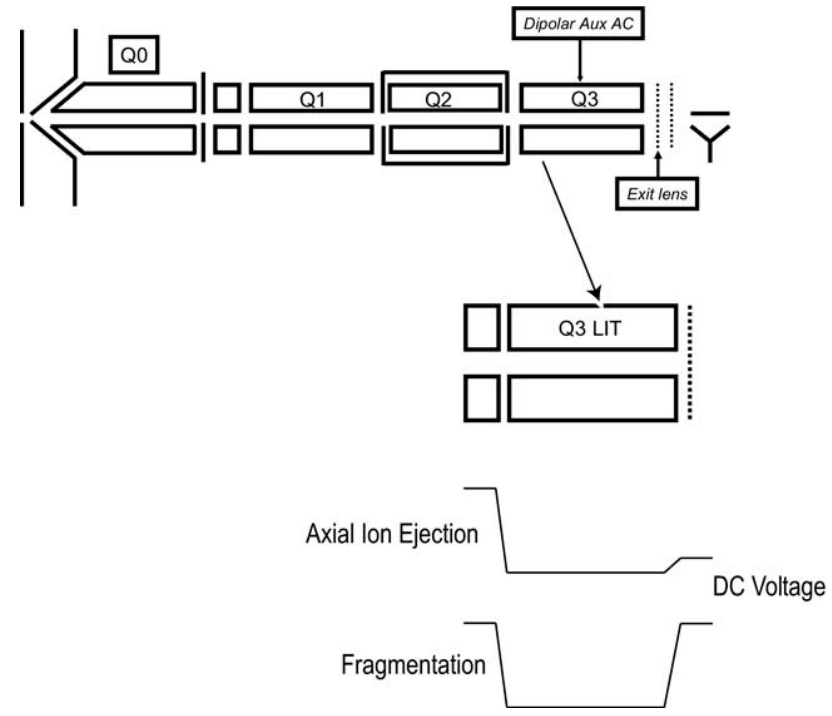

Figure 1. A schematic of the experimental apparatus that shows an expanded view of the Q3 linear ion trap. In the ejection and fragmentation experiments, ions are mass-selected at unit resolution using the Q1 quadrupole and trapped in the Q3 LIT. For nonresonant axial ejection, the exit lens voltage is only a few volts repulsive. For fragmentation, the exit lens voltage is about $100 \mathrm{~V}$ repulsive during excitation. A mass analysis scan of the LIT is conducted following CID.

frequency modulations. Because of this, all temporal profiles shown here were acquired with the excitation field phase set to $0^{\circ}$.

MSAE mass spectra were generated using a dipolar frequency of $310 \mathrm{kHz}(q \sim 0.84)$ and amplitudes of $<1$ $\mathrm{V}_{\mathrm{p}-\mathrm{p}}$ delivered through a center-tapped bipolar toroidal transformer. Off-resonance excitation was performed at $q$-values from 0.2 to 0.8 and excitation amplitudes up to $6 \mathrm{~V}_{\mathrm{p}-\mathrm{p}}$. The pressure within the linear ion trap could be adjusted over the range of $1.5-5.5 \times 10^{-5}$ torr.

Electrospray-generated ions traveled through a curtain gas and differential pumping regions into an RFonly quadrupole ion guide $(\mathrm{Q} 0)$ within a chamber maintained at about $8 \times 10^{-3}$ torr, through the Q1 quadrupole mass filter, and the gas-filled collision cell at low energy $(<10 \mathrm{eV})$, and into the Q3 linear ion trap. Two lenses were located downstream of Q3: the first, a mesh-covered 8-mm aperture; the second, a clear 8-mm diameter aperture. The mesh-covered lens is referred to as the exit lens and the clear aperture lens is referred to as the deflector.

There were two basic scan functions used in these studies: one to generate the temporal axial ion ejection data and the other for fragmentation following offresonance excitation. The time-resolved data were collected in the following manner.

- The most intense isotope was isolated upstream using the Q1 RF/DC quadrupole and introduced into the Q3 linear ion trap.

- The ion population was then cooled for $100 \mathrm{~ms}$.

- After this thermalization step, the exit lens was adjusted, from 100 to $2-5 \mathrm{~V}$ repulsive. 
- The drive RF voltage applied to the linear ion trap was jumped to the off-resonance excitation point at which time the auxiliary AC field was turned on and ion detection carried out for 20 to $50 \mathrm{~ms}$.

This scan function was repeated and averaged approximately 100 times to produce the data shown here.

The second scan function was used to generate product ion mass spectra following excitation using an off-resonant field. The major difference with the aforementioned scan function involves the magnitude of the exit lens barrier at the end of the linear ion trap during the off-resonance excitation step.

- The most intense isotope was isolated upstream using the Q1 RF/DC quadrupole and introduced into the linear ion trap.

- The ion population was then cooled for $100 \mathrm{~ms}$.

- Following the thermalization step, the exit lens was maintained $100 \mathrm{~V}$ repulsive, the drive RF voltage was adjusted to the appropriate value, and the auxiliary off-resonant field was applied for a predetermined amount of time.

- The ions within the linear ion trap were then stored for an additional $70 \mathrm{~ms}$.

- The trapped ion population was scanned out of the linear ion trap in the usual manner, generating a product-ion spectrum.

Ions were detected via an ETP (Sydney, Australia) discrete dynode electron multiplier, operated in pulsecounting mode, with the entrance floated to $-6 \mathrm{kV}$ for positive ions.

The prototype instrument control and data acquisition system allowed for a minimum dwell time of 2.5 $\mu \mathrm{s}$. The modulation frequencies were obtained using the DPLOT Fast Fourier Transform program (HydeSoft Computing, Vicksburg, MS, USA).

Reserpine (100 pg/ $\mu \mathrm{L}, 50 / 50$ water/acetonitrile with $0.1 \%$ acetic acid) and a $1 / 100$ dilution of an Agilent electrospray tuning solution (Agilent ES tuning mix, G2421) were introduced via a pneumatically assisted electrospray ion source.

\section{Results and Dicussion}

\section{On-versus Off-Resonance Ion Excitation and Ejection}

To a first approximation the secular frequency, $\omega_{0}$, of an ion confined in an RF-only linear ion trap is given by the following expression:

$$
\omega_{0}=\beta \frac{\Omega}{2} \approx q \frac{\Omega}{\sqrt{8}}=\frac{4 e V}{m \Omega^{2} r_{0}^{2}} \frac{\Omega}{\sqrt{8}},
$$

where $2 r_{0}$ is the shortest distance between opposing rods, $e$ is the electronic charge on the ion of mass $m, q$ is the Mathieu stability coordinate, $\Omega$ is the angular fre- quency, and $V$ is the amplitude of the drive RF voltage. In a perfect quadrupole, on-resonance excitation from an auxiliary dipolar field leads to a linear increase in radial amplitude until the excited ion undergoes CID or is neutralized on the trap electrodes [8]. The linear ion trap used here is fabricated with round electrodes and, as such, has contributions from higher-order multipole fields [8-10]. The effect of these higher-order fields is to introduce a degree of radial dependence to the ion's secular frequency. An ion initially excited from the center of the linear ion trap will gain radial amplitude, but will gradually go out-of-resonance with the excitation field and begin to return to the center line of the ion trap, at which point it will be re-excited, thus beginning the process over again. This phenomenon has been exploited to obtain high-efficiency CID in a low-pressure linear ion trap [8].

MSAE from a linear ion trap arises from the fact that the radial RF fields diminish at the ends of the device, creating a radial position-dependent axial field in the ion trap fringing fields as shown in the following expression [4]:

$$
\left\langle E_{\mathrm{z}, \text { quad }}\right\rangle_{\mathrm{RF}}=\left|\frac{\partial f(z)}{\partial z}\right| \frac{m \Omega^{2}}{8 e} q^{2}\left(X^{2}+Y^{2}\right),
$$

where $X^{2}+Y^{2}$ is the square of the radial displacement attributed to secular motion and $f(z)$ describes the exit fringing field.

It has been shown [4] that there is a net positive axial electric field out of the linear ion trap. Equation 3 illustrates that, to a first approximation, the axial electric field out of the linear ion trap is proportional to the square of the radial displacement, the square of the $q$-value, and the mass-to-charge ratio of the trapped ion, and is also independent of the exact form of the fringing field, $f(z)$.

Within the context of the current work, both on- and off-resonance excitation leading to MSAE require a buildup of radial amplitude sufficient to overcome the repulsive DC field at the exit end of the linear ion trap. The auxiliary dipolar field provides the radial excitation. Examples of temporal profiles of ion ejection following on- and off-resonance ion excitation and mass-selective ion ejection are shown in Figure 2. For an on-resonance field, as shown in Figure $2 \mathrm{a}$, the excited ions are ejected promptly. The width of the profile is largely determined by the excitation amplitude. Increasing amplitudes lead to more prompt and narrower temporal envelopes.

The ejected ion temporal profiles during off-resonance excitation are qualitatively different from the onresonance excitation case, as illustrated in Figure $2 b-d$. Under the appropriate excitation amplitude conditions, a periodic beat pattern is observed on the ejected ion temporal profiles that can extend for tens of milliseconds. Again, the duration of the temporal profile is largely determined by the amplitude of the auxiliary excitation field. At high excitation amplitudes fewer 

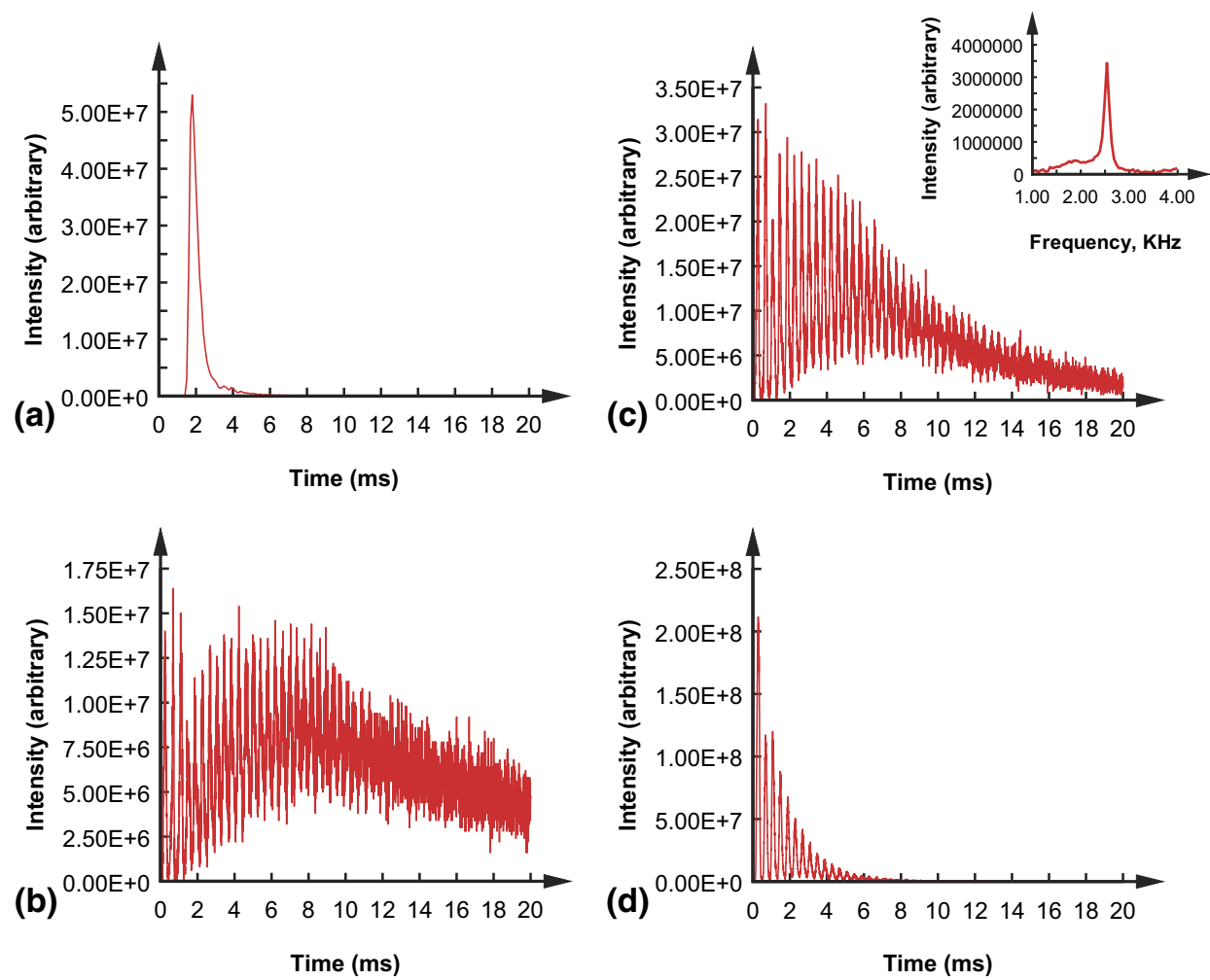

Figure 2. The temporal profiles of the axial ejection of the protonated reserpine ion at $\mathrm{m} / \mathrm{z} 609$ under (a) on-resonance conditions at a $q$-value of 0.65 and an amplitude of $12 \mathrm{mV}$, and $2.54 \mathrm{kHz}$ blue-shifted off-resonance excitation at (b) $2600 \mathrm{mV}$, (c) $3140 \mathrm{mV}$, and (d) $4600 \mathrm{mV}$. The exit barrier was $5 \mathrm{~V}$ repulsive. The FFT of $(\mathbf{c})$ is shown in the inset.

beats are detected because the higher excitation field depletes the trapped ion population more quickly. At the lower amplitude of the off-resonance excitation voltage used in Figure $2 b$, a gradual increase of ejected ion current can be seen that is presumably the result of a corresponding gradual increase in radial excursion of the excited ion population with time. This buildup of ejected ion signal corresponds to increasing radial amplitude with increasingly long exposure to the offresonance field. In Figure $2 b$, the maximum ejected ion intensity occurs after about $7 \mathrm{~ms}$ of excitation. Higheramplitude off-resonance excitation fields are characterized by a more prompt buildup of radial amplitude, as can be observed in Figure 2c and d.

The detected ion signal decay in the ejected temporal profile is a result of the loss of the trapped ion population because of a variety of different processes, including ejection toward the detector, neutralization on the linear ion trap electrodes, and fragmentation. At high excitation amplitudes, axial ejection toward the detector and neutralization on the ion trap electrodes dominate all other processes. At lower amplitudes, ejection toward the detector is the dominant loss process with ejection efficiencies measured to be $>60 \%$. As the exit barrier is made more repulsive, the excited ion population continues to gain more radial amplitude to the point at which sufficient internal energy is deposited to produce fragment ions.
The beat pattern on the ejected ion temporal profiles is, to a first approximation, a measure of the degree of detuning from the secular frequency. Figure 3 displays a plot of measured beat frequency versus detuning for excitation at $q=0.75$. There is a nearly $1: 1$ correspondence between the degree of detuning and the measured beat frequencies. This suggests that measurements of the beat patterns on the ejected ion temporal profiles can provide reasonably accurate measures of

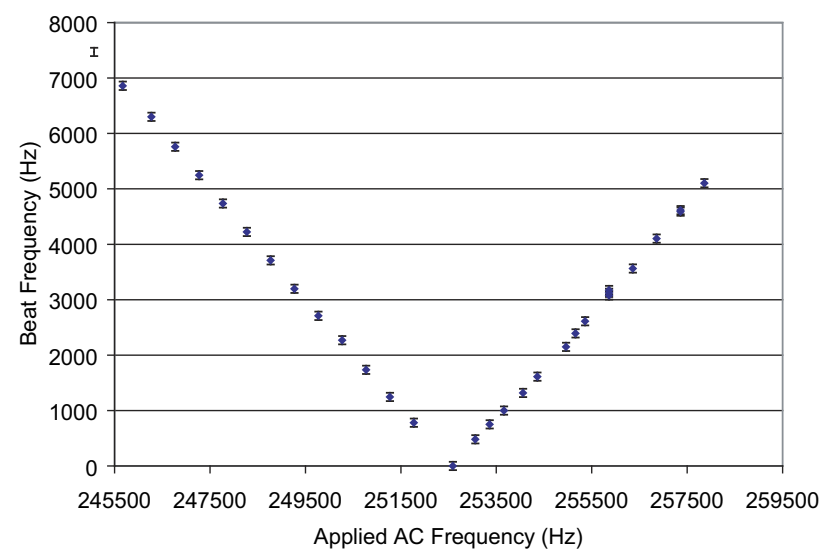

Figure 3. The relationship between measured beat frequency and the frequency of the off-resonance AC field near $q=0.74$ of the protonated reserpine ion at $\mathrm{m} / \mathrm{z} 609$. 
the ions' secular frequencies. To date measurements of trapped ion secular frequencies in linear ion traps with axial ejection are most often accomplished using the "depletion method," in which a mass-selected trapped ion population is resonantly excited to the point of fragmentation or neutralization on the trap electrodes $[8,10]$. As such, one can consider this experiment as a measure of the entire range of radial amplitudes that the ions can experience from near $r \sim 0$ to near $r_{0}$. Secular frequencies measured using the depletion method can differ from those measured under MSAE experiments because the exit DC barrier is much greater in the depletion/fragmentation experiments, leading to differing trapping forces. In addition the trapped ions are driven to much greater radial amplitudes in the depletion/fragmentation studies, making the contribution of higher-order multipole fields more important than those in the MSAE experiments. The current technique provides a method for measurement of ion secular frequencies in which a smaller range of radial amplitudes is explored. In MSAE, once a trapped ion is resonantly excited, it is the magnitude of the exit barrier height that determines the maximum radial excursion [4]. Changes in the beat frequency as a function of changes of trapping forces correspond directly to changes in ion secular frequency. This is a much more relevant approach to gaining understanding about characteristics of ion motion that is important for MSAE.

With increased time resolution for the detected ion current and using a fixed-phase excitation field, additional higher-frequency components become apparent on the ejected ion temporal profiles, as illustrated in Figure 4. The beat frequency that is prominent in both Figure $4 \mathrm{a}$ and $\mathrm{b}$ corresponds to the detuning from the secular frequency, as discussed earlier, and is visible at $3.5 \mathrm{kHz}$. There is also a weaker, higher-frequency modulation observed in Figure $4 \mathrm{~b}$ at the excitation frequency of $83.6 \mathrm{kHz}$. This is driven, coherent motion of the ion population by the off-resonance field. The fact that these high-frequency modulations are not observed in the temporal profile shown in Figure $4 \mathrm{a}$ is a result of the signal averaging of the random-phase excitation source, which wash out the high-frequency modulations. The data in Figure $4 \mathrm{~b}$ were obtained when the arbitrary waveform generator used as the excitation source was operated in "burst mode," which preserves the phase relationship for repetitive triggering events. This preserves the higher-frequency modulations.

Figure 5 illustrates the effects of termination of the off-resonance excitation field at different phases of the ion motion. The top trace shows the ejected ion temporal profile when the excitation field is terminated after $1200 \mu \mathrm{s}$ (100 cycles of the excitation field). There is no significant detected ion current after the termination of the excitation field, which implies that the ions are no longer radially excited and that the field was terminated at, or near, a radial amplitude minimum.
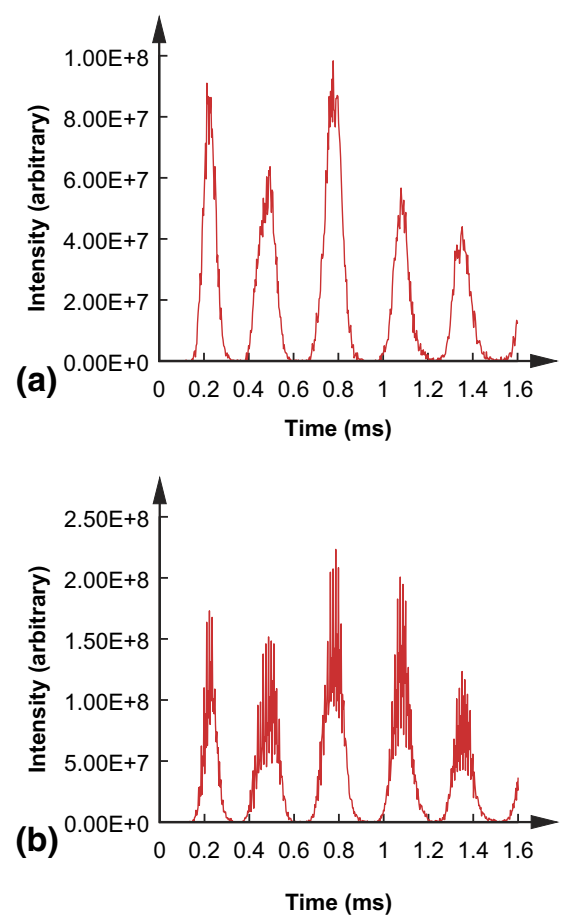

Figure 4. A comparison of the early portions of the temporal ejection profiles of the protonated $\mathrm{m} / \mathrm{z} 622$ ion from the Agilent ESI tuning mix and a $q$-value of about 0.28 under (a) random phase off-resonance excitation conditions and (b) constant phase offresonance excitation conditions. The beat frequency in both profiles is $3.5 \mathrm{kHz}$. The higher frequency component apparent in (b) corresponds to the frequency of the excitation field at $83.6 \mathrm{kHz}$. The combination of signal averaging and an excitation field with a random phase washes out the higher frequency component in the ejection profile shown in (a).

In contrast, terminating the excitation field at a radial amplitude maximum, as shown in Figure 5b, leaves the ions with significant kinetic energy. Here, the offresonance excitation field was terminated after $86 \mathrm{cy}-$ cles, or $1030 \mu \mathrm{s}$. The subsequent ejected ion time evolution profile is characterized by a general exponential decay of the detected ion signal with a time constant of about $3.5 \mathrm{~ms}$. The time constant of this decay is mainly a function of the pressure within the linear ion trap and the amplitude of the off-resonance excitation signal. Without the off-resonance excitation field, the beat structure decays away in about $1 \mathrm{~ms}$.

The high-frequency modulations decay at approximately the same rate as the overall ejected ion signal. The frequency of this ion motion, however, has changed from that of the off-resonance excitation field at 83.6 $\mathrm{kHz}$ to the ions' secular frequency at $87.1 \mathrm{kHz}$. A close examination of the temporal profile of the ejected ion signal at the point at which the excitation field is terminated shows that the change in frequency from that of the driving field to the true secular frequency occurs very quickly. All measurements to date indicate that the frequency change occurs on the same timescale as the fall time of the AC field itself, which is $<50 \mu \mathrm{s}$.

The ejected ion profiles are also expected to have a modulation at the frequency of the micromotion of the 

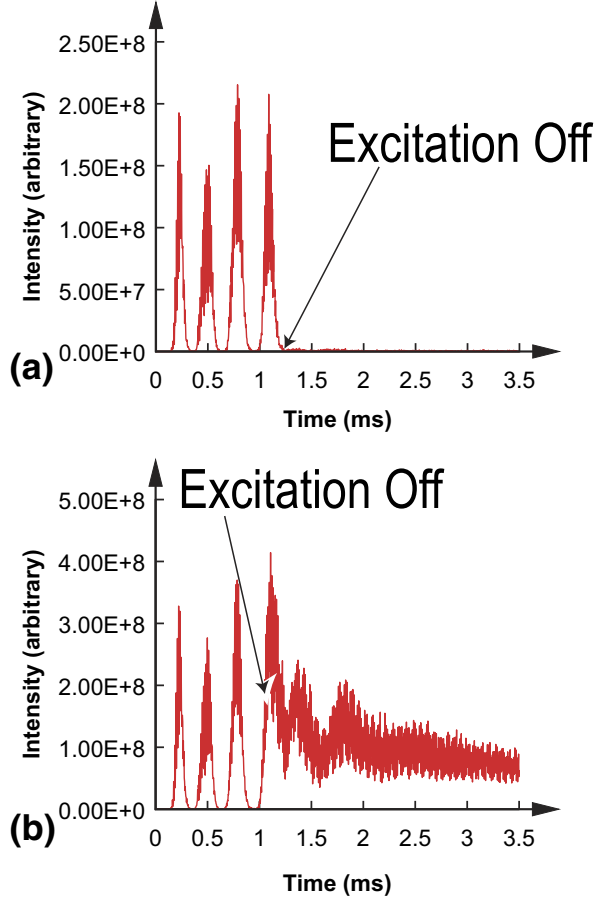

Figure 5. The effects of termination of the off-resonance excitation field at two different time points on the temporal profiles of the axial ejection of the $\mathrm{m} / \mathrm{z} 622$ ion from the Agilent tuning mix at $3.5 \mathrm{kHz}$ red-shifted at an excitation frequency of $83.6 \mathrm{kHz}$. The profile in (a) demonstrates the result of terminating the excitation field after 100 cycles of the excitation frequency $(1200 \mu \mathrm{s})$, whereas (b) shows the result of excitation field termination after 86 cycles of the excitation frequency $(1030 \mu \mathrm{s})$. The higher-frequency modulations in (b) change from that of the excitation field at $83.6 \mathrm{kHz}$ to the secular frequency at $87.1 \mathrm{kHz}$ on termination of the off-resonance field.

trapped ions at the RF drive frequency [4]. Unfortunately, the time resolution of the current detection is insufficient to resolve modulations at $0.816 \mathrm{MHz}$.

\section{Off-Resonance Excitation for Fragmentation}

The preceding discussion has demonstrated that a selectable amount of radial kinetic excitation can be imparted through proper choice of the termination point of an off-resonance excitation field during the beat cycle. This radial excitation can be used for collisional activation, leading to fragmentation. Ions that are left with significant radial excitation will continue to experience collisions with background neutrals leading to collisional activation that can lead to dissociation. Figure 6 shows the results of a series of experiments in which a trapped ion population has been excited in an off-resonance manner for different periods of time. Each data point corresponds to a signal-averaged production spectrum in which the off-resonance excitation field has been active for a fixed number of AC cycles. The excitation step was followed by an ion storage period of $70 \mathrm{~ms}$ in which any sufficiently activated ions were allowed to dissociate. Finally, all trapped ions were scanned out of the Q3 linear ion trap and detected. Two representative product-ion spectra are shown in Figure 6 displaying the dramatic difference.

Figure 6 is remarkably similar to results from SORI experiments in an FT-ICR [11]. The lower-pressure FT-ICR work exhibits timescales that are nearly 10 -fold longer, which is not surprising considering the roughly 10 -fold lower pressure regime for the pulsed gas ICR work.

The measured fragmentation efficiencies are roughly $60 \%$ in this example, which is about the same level expected from on-resonance excitation at the same $q$-value. It is noteworthy to point out that CID efficiency in the linear ion trap does not seem to be a function of the degree of detuning of the off-resonance field from the ions' secular frequency, which is in contrast to off-resonance excitation in an FT-ICR [1].

Because of the short excitation durations associated with the product-ion spectra in Figure 6, most of the dissociation occurs after the off-resonance excitation field has been terminated. The illustration of offresonance excitation for as little as $146 \mu \mathrm{s}$, leading to efficient fragmentation, suggests that dynamic CID techniques such as "fast excitation CID" [12] and similar approaches $[13,14]$ might benefit from the offresonance approach.

Although not shown here, there seems to be a complicated relationship between fragmentation efficiencies, excitation $q$-value, and red- or blue-shifted excitation fields. These relationships are most likely explained by the nature of the higher-order multipole fields in these round-rod linear ion traps. This will be the subject of future investigations.

\section{Conclusions}

A method for observation of the dynamics of ion clouds stored in a low-pressure linear ion trap has been presented. When coupled with mass-selective axial ejection clear modulations of the temporal profiles of the ejected ion signal that correspond to the beat frequency are observed. The modulations are prominent because of the very strong dependence of the axial ejection process on radial amplitude. This approach has enabled observation of the buildup of radial excitation with increasing excitation time when using low-amplitude excitation fields. It also provides a new way of measuring the secular frequency of the trapped ions by using the measured beat frequency and the known excitation frequency. Since these secular frequencies are determined from ejected ion signal rather than through depletion experiments, they should more closely reflect the trapping forces and radial excursions during a mass-selective axial ejection scan. The ability to determine characteristic frequencies under realistic ion trap scan will be useful in understanding some of the subtleties of the influences of trapping forces on the performance of MSAE mass spectrometers. 


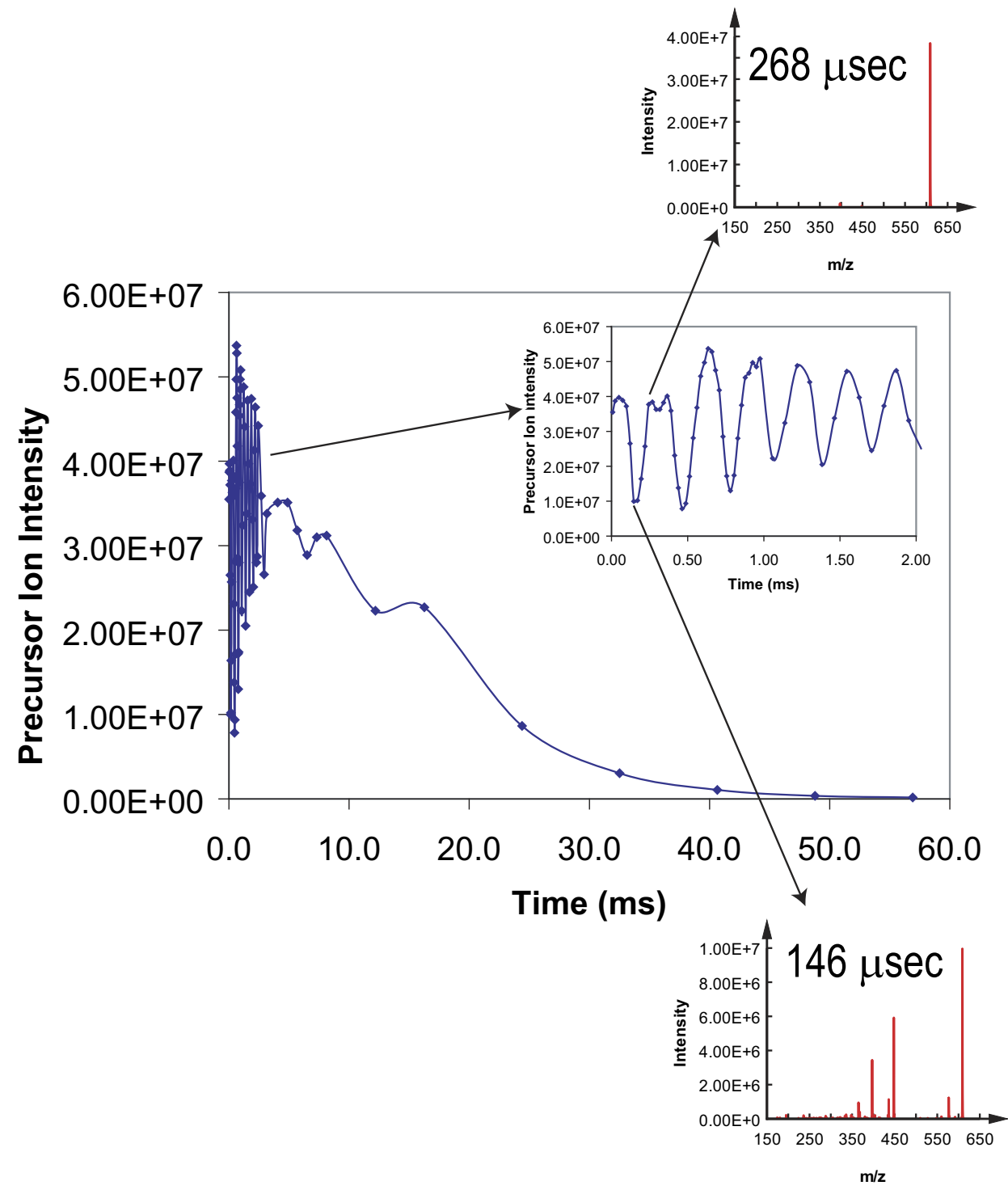

Figure 6. A plot of the intensity of the residual protonated reserpine ion at $\mathrm{m} / \mathrm{z} 609$ versus off-resonance excitation time. Off-resonance excitation was conducted at a frequency of $123.0 \mathrm{kHz}$, which corresponded to a red-shifted detuning of $3.4 \mathrm{kHz}$ and a $q$-value of about 0.34 . The inset displays an expanded view of the early time points. Two of the resultant product ion spectra corresponding to the time points $146 \mu \mathrm{s}$ (18 cycles of the excitation frequency) and $268 \mu \mathrm{s}$ (33 cycles of the excitation frequency) are also shown. The precursor ion intensity clearly follows the $3.4 \mathrm{kHz}$ beat pattern.

Termination of the off-resonance excitation field at the appropriate point in the beat cycle can leave the ion packet with essentially a user-selectable degree of radial excitation. One can envision the possibility of radially exciting a trapped ion population for tens to hundreds of microseconds and then returning them to a point of low excitation from which the dissociation dynamics can be monitored. The degree of collisional excitation can be controlled by the beat frequency, the number of excitation beats, and the amplitude of the excitation field in addition to the Mathieu $q$-value. This approach may allow better control of the precise amount of internal energy deposited into a collisionally activated ion.
Finally, the ability to visualize the manifestations of the low-frequency beats, the higher-frequency-driven motion at the excitation frequency, and the secular ion motion makes it possible to explore the dephasing dynamics as a function of a variety of linear ion trap parameters, including collision frequency, $q$-value, and ion number density. Higher temporal resolution should provide a glimpse of the micromotion at the RF frequency.

\section{Acknowledgments}

The author thanks Frank Londry for helpful discussions on various topics of ion excitation dynamics. 


\section{References}

1. Laskin, J.; Futrell, J. H. Activation of Large Ions in FT-ICR Mass Spectrometry. Mass Spectrom. Rev. 2005, 24, 134-167.

2. Qin, J.; Chait, B. T. Matrix-Assisted Laser Desorption Ion Trap Mass Spectrometry: Efficient Isolation and Effective Fragmentation of Peptide Ions. Anal. Chem. 1996, 68, 2108-2112.

3. Qin, J.; Chait, B. T. Collision-Induced Dissociation of Singly Charged Peptide Ions in a Matrix-Assisted Laser Desorption Ionization Ion Trap Mass Spectrometer. Int. J. Mass Spectrom. 1999, 190/191, 313-320.

4. Londry, F. A.; Hager, J. W. Mass Selective Axial Ion Ejection from a Linear Quadrupole Ion Trap. J. Am. Soc. Mass Spectrom. 2003, 14, 1130-1147.

5. Oomans, J.; von Helden, G.; Meijer, G. Experimental Observation of Laser-Induced Coherent Ion Motion in a Quadrupole Ion Trap. Int. J. Mass Spectrom. 2000, 221, 163-176.

6. Weil, C.; Wells, J. M.; Wollnik, H.; Cooks, R. G. Axial Ion Motion within the Quadrupole Ion Trap Elucidated by DC Pulse Tomography. Int. J. Mass Spectrom. 2000, 194, 225-234.

7. Plass, W. R.; Gill, L. A.; Bui, H. A.; Cooks, R. G. Ion Mobility Measurement by DC Tomography in an RF Quadrupole Ion Trap. I. Phys. Chem. A 2000, 104, 5059-5065.
8. Collings, B. A.; Stott, W. R.; Londry, F. A. Resonant Excitation in a Low-Pressure Linear Ion Trap. J. Am. Soc. Mass Spectrom. 2003, 14, 622-634.

9. Douglas, D. J.; Glebova, T. A.; Konenkov, N. V.; Sudakov, M. Y. Spatial Harmonics of the Field in a Quadrupole Mass Filter with Circular Electrode. Tech. Phys. 1999, 44, 1215-1219.

10. Douglas, D. J.; Frank, A. F.; Mao, D. Linear Ion Traps in Mass Spectrometry. Mass Spectrom. Rev. 2005, 24, 1-29.

11. Schnier, P. D.; Jurchen, J. C.; Williams, E. R. The Effective Temperature of Peptide Ions Dissociated by Sustained Off-Resonance Irradiation Collisional Activation in Fourier Transform Mass Spectrometry. J. Phys. Chem. B 1999, 103, 737-745.

12. Murrell, J.; Despeyroux, D.; Lammert, S. A.; Stephenson, J. L.; Goeringer, D. E. "Fast Excitation CID" in a Quadrupole Ion Trap Mass Spectrometer. J. Am. Mass Spectrom. 2003, 14, 785-789.

13. Schwartz, J. C.; Syka, J. P.; Quarmby, S. T. Improving the Fundamentals of MSN on 2D Ion Traps: New Ion Activation and Isolation Techniques. Proceedings of the 53rd ASMS Conference on Mass Spectrometry; San Antonio, TX, June 2005.

14. Cunningham, C.; Glish, G. L.; Burinsky, D. J. High Amplitude Short Time Excitation: A Method to Form and Detect Low Mass Product Ions in a Quadrupole Ion Trap Mass Spectrometer. J. Am. Soc. Mass Spectrom. 2006, 17, 81-84. 